\title{
Hybrid Viabahn-Assisted Bypass for Long Femoro-Popliteal Occlusive Disease \\ - Midterm Results -
}

\author{
Po-Lin Chen, MD; Ting-Chao Lin, MD; I-Ming Chen, MD, PhD
}

\begin{abstract}
Background: Endovascular therapy has become the first-line treatment for most peripheral artery diseases, but re-entry into the true lumen is not always possible, particularly in some cases of chronic total occlusion (CTO). We previously reported a novel hybrid Viabahn-assisted bypass (VAB) technique to facilitate revascularization of the femoro-popliteal CTO. This study assessed the midterm results of $\mathrm{VAB}$ technique.
\end{abstract}

Methods and Results: Between January 2013 and April 2017, 440 patients received femoro-popliteal intervention and 17 of them (mean age, 76.2 years; 11 male patients) were treated using the VAB technique. The technical success was $100 \%$. The mean follow-up period was $27.4 \pm 14.4$ months (range, 6-50 months). Two patients underwent major amputation at 6 and 11 months postoperatively, but they died of acute myocardial infarction and of sepsis, respectively. Another 3 patients received re-intervention because of edge stenosis of the Viabahn stent graft. Another patient had acute Viabahn thrombosis and was treated using open thrombectomy. The primary and secondary patency rates at 12 months were both $88.2 \%$, and at 24 months they were $69.2 \%$ and $84.6 \%$, respectively.

Conclusions: The VAB technique could be a safe and feasible alternative treatment for long femoro-popliteal CTO when conventional treatments fail. The technical success is high, and the midterm patency rates are acceptable.

Key Words: Endovascular therapy; Femoro-popliteal artery; Peripheral arterial disease; Viabahn-assisted bypass

$\mathbf{O}$ wing to advancements in technology and improved outcomes in recent years, endovascular therapy (EVT) is now widely used as a first-line treatment for complex peripheral artery disease (PAD) instead of traditional open bypass surgery. ${ }^{1-3}$ The use of the Viabahn (W.L. Gore, Flagstaff, AZ, USA) covered stent, 1 possible EVT, has shown favorable long-term results for treatment of TransAtlantic Inter-Society Consensus II (TASC II) ${ }^{4} \mathrm{C}$ or D femoropopliteal (FP) steno-occlusive disease, ${ }^{1,2,5-7}$ and the results are similar to those of surgical revascularization using the conventional femoral-to-above-knee popliteal artery bypass method with a synthetic graft. ${ }^{\mathbf{8}, 9}$

Wiring through an entire FP chronic total occlusion (CTO) using intraluminal, subintimal, or subintimal arterial flossing with antegrade-retrograde intervention (SAFARI) ${ }^{10}$ is responsible for the success of EVT. In the case of severely calcified occlusive lesions, however, wiring may not be achievable despite the use of various endovascular techniques, thereby causing EVT failure. The hybrid Viabahn-assisted bypass (VAB) technique, which combines small open surgery and the SAFARI technique, ${ }^{11}$ is designed for cases in which bypass surgery is unsuitable and EVT failure is certain. The VAB technique improves the success rate of FP revascularization without requiring any special device.

The aim of the present study was therefore to report the midterm results of VAB for long superficial femoral artery (SFA) occlusive lesions at Taipei Veterans General Hospital, Taiwan.

\section{Study Design}

A retrospective review of 17 consecutive patients who underwent attempted recanalization of SFA CTO using the VAB technique between January 2013 and April 2017 was conducted. The institutional review board at Taipei Veterans General Hospital, Taiwan, approved this retrospective study, and the need for informed consent was waived because of the retrospective nature of this study. All patients had critical limb ischemia with rest pain or tissue loss (Rutherford categories 4-6), and all preoperative computed tomography angiography (CTA) showed long SFA CTO lesions $(>15 \mathrm{~cm})$. Vascular surgeons with a

Received February 5, 2018; revised manuscript received March 25, 2018; accepted April 18, 2018; released online May 29 , 2018 Time for primary review: 30 days

Division of Cardiovascular Surgery, Department of Surgery, Taipei Veterans General Hospital, Taipei (P.-L.C., I.-M.C.); Department of Medicine, School of Medicine, National Yang-Ming University, Taipei (P.-L.C., T.-C.L., I.-M.C.); and Heart Center, Cheng-Hsin Rehabilitation Medical Center, Taipei (T.-C.L.), Taiwan

Mailing address: I-Ming Chen, MD, PhD, Division of Cardiovascular Surgery, Department of Surgery, Taipei Veterans General Hospital, No. 201, Sec. 2, Shipai Road, Beitou District, Taipei City, Taiwan. E-mail: alomar2099@gmail.com

ISSN-1346-9843 All rights are reserved to the Japanese Circulation Society. For permissions, please e-mail: cj@j-circ.or.jp 
similar training background and a high level of consensus regarding treatment policies owing to routine periodical meetings performed all the revascularization procedures. EVT was considered the first-line treatment if the patients were not eligible for bypass surgery owing to high anesthesia-associated risk or the unavailability of great saphenous vein (GSV) graft. The hybrid VAB technique was used when endovascular wire cannulation of the SFA CTO could not be achieved.

\section{VAB}

A detailed description of the VAB technique has been provided previously. ${ }^{11}$ The antegrade wire was directed from the common femoral artery either contralaterally or ipsilaterally. An intraluminal approach was attempted first, followed by subintimal recanalization. If the antegrade approach failed, we switched to a retrograde approach. Direct puncture of the distal SFA, popliteal artery, anterior tibial artery, or posterior tibial artery was performed using ultrasound or fluoroscopy guidance. Retrograde recanalization was attempted intraluminally or in combination with the SAFARI technique (Figure 1A,B). If the antegrade and retrograde approaches both failed, VAB was used. Using fluoroscopy guidance, a $3-\mathrm{cm}$ incision was made immediately above the middle SFA where the antegrade and retrograde guidewires overlapped. A longitudinal or transverse arteriotomy was made without transection. Both the antegrade and retrograde guidewires were retrieved with the supporting catheter from the antegrade access (Figures 1C,2A). The antegrade guidewire was removed, and the retrograde guidewire was manually inserted into the antegrade supporting catheter (Figure 2B) and externalized from the antegrade access as the flossing wire (Figures 1D,2C). Predilatation was performed using the antegrade approach and then a Viabahn was deployed across the arteriotomy site (Figures 1E,2D). Postdilatation was performed, and the restoration of the blood flow was confirmed on angiography. After hemostasis was achieved, the wound was closed using the conventional method.

\section{Medication and Follow-up Protocol}

All patients received $75 \mathrm{mg}$ clopidogrel per day for $\geq 1$ year if it was not contraindicated. Follow-up included symptom evaluation every 3 months at an outpatient department, survey of the ankle-brachial index (ABI) every 6 months, and lower limb CTA postoperatively at 6 and 12 months and then once yearly.

\section{Study Endpoint and Definition}

Technical success was defined as the successful deployment of the Viabahn stent graft and restoration of distal blood flow. The primary outcomes included technical success, Viabahn patency, PAD-related death, major amputation (above- or below-knee amputation), and ABI.

\section{Statistical Analysis}

Parameters were analyzed using SPSS 18.0 (SPSS Inc. Chicago, IL, USA). Continuous variables are expressed as mean \pm SD. Wilcoxon signed rank test was used to compare the postoperative and preoperative parameters. Patency rates were determined using Kaplan-Meier analysis.

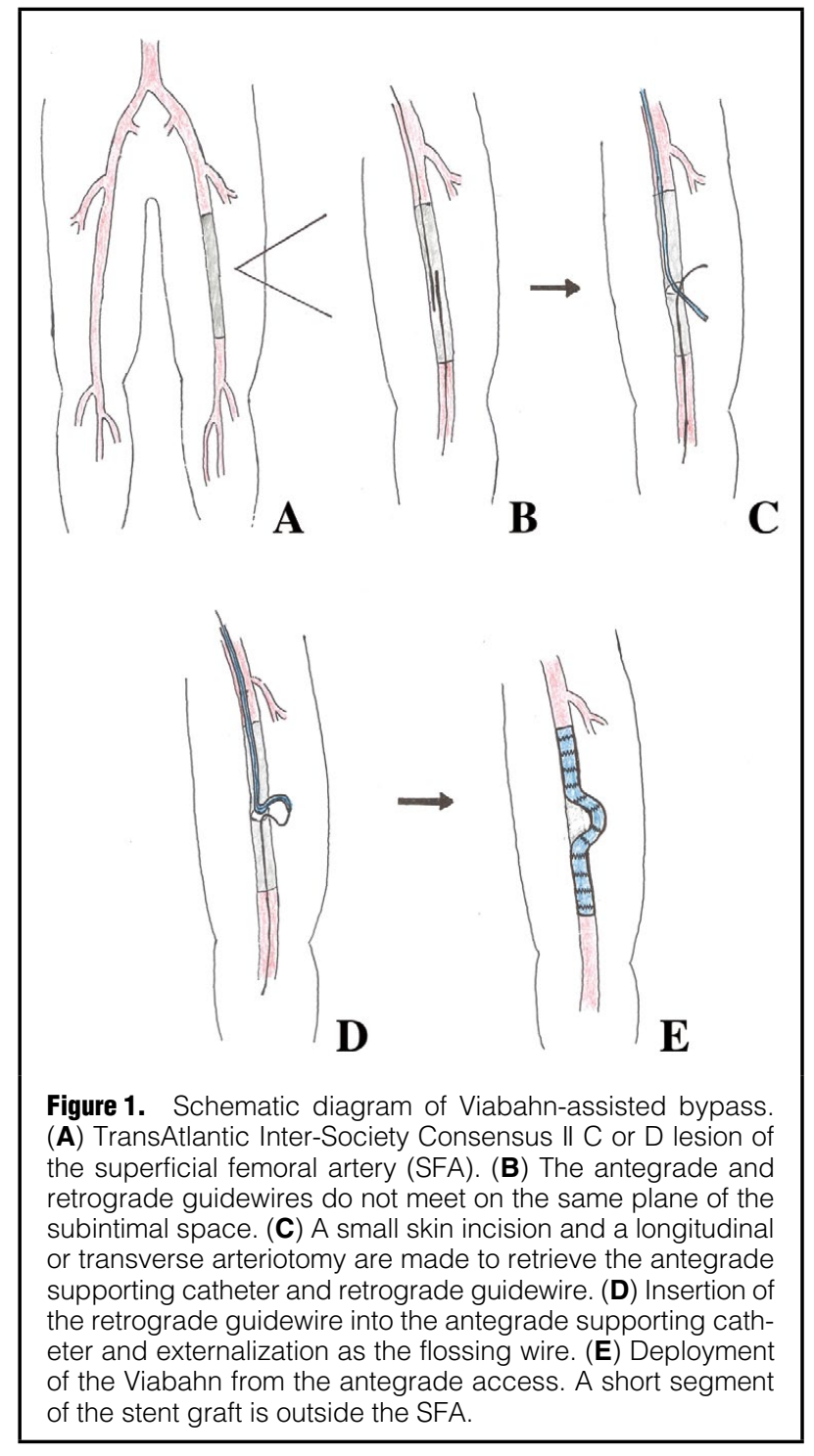

\section{Results}

Procedure Results

The demographic features and comorbidities of the 17 patients (mean age, 76.2 years; 11 male patients), are listed in Table 1. Technical success was achieved in all patients. Procedural data are listed in Table 2. All patients received 2 Viabahn stent grafts, except for 1 patient who received 3 stent grafts. Fourteen patients had 2 distal run-offs, and 3 had only 1 run-off. Thirteen patients received below-theknee (BTK) interventions, and 5 patients received concomitant iliac stenting. One patient had a contralateral iliac artery rupture with a retroperitoneal hematoma caused by short-segment subintimal wire passage. The patient required iliac stent grafting to stop the bleeding. The other patients did not have intraoperative complications.

\section{Midterm Results}

All 17 patients were followed up according to the study protocol, and no patients were lost to follow-up. The mean follow-up was $27.4 \pm 14.4$ months (range, 6-50 months). All the endpoint outcomes are listed in Table 3. Procedure- 


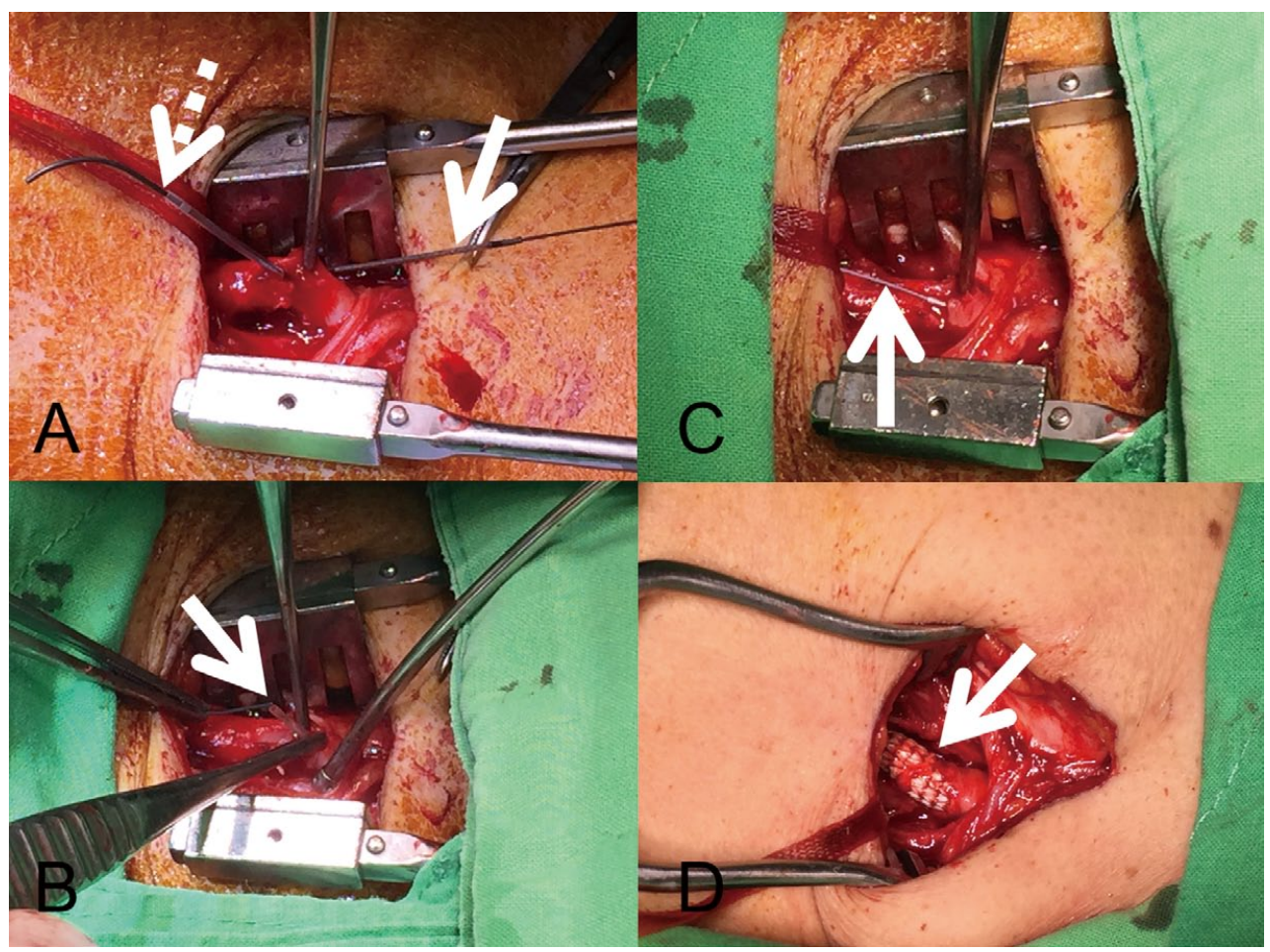

Figure 2. Viabahn-assisted bypass technique with open subintimal arterial flossing antegrade and retrograde intervention and covered stent implantation. (A) After a small incision is made to expose the middle of the superficial femoral artery (SFA) and longitudinal arteriotomy is performed, the guidewire and supporting catheter from the (white dotted arrow) antegrade and (white arrow) retrograde access are both retrieved. (B) White arrow, manual insertion of the retrograde guidewire into the antegrade catheter. (C) The flossing guidewire (white arrow) is established and spans the arteriotomy of the middle SFA. (D) A covered stent (white arrow) is then deployed to cover the lesion, and is positioned across the arteriotomy of the middle SFA.

\begin{tabular}{|lc|}
\hline Table 1. Patient Demographics & Mean \pm SD or $\mathbf{n}$ \\
& $76.2 \pm 5.8$ \\
Age (years) & 11 \\
Male & 11 \\
Smoking & 10 \\
Hypertension & 12 \\
Diabetes mellitus & 10 \\
Hyperlipidemia & 10 \\
CAD & 8 \\
Carotid stenosis $>70 \%$ & 4 \\
Stroke & 9 \\
Hemodialysis & $0.51 \pm 0.1$ \\
Preoperative ABI & $4.7 \pm 0.8$ \\
Rutherford class & $19.7 \pm 4.0$ \\
SFA lesion length $(\mathrm{cm})$ & \\
\hline
\end{tabular}

$\mathrm{ABI}$, ankle-brachial index; $\mathrm{CAD}$, coronary artery disease; SFA, superficial femoral artery.

related mortality was not observed. One patient died of acute myocardial infarction 6 months postoperatively. That patient had newly developed renal failure and received hemodialysis. Another patient died of sepsis at 11 months postoperatively. Both patients received additional percutaneous transluminal angioplasty (PTA) for BTK

\begin{tabular}{|lc|}
\hline Table 2. Procedure Data & Mean \pm SD or $\mathbf{~}$ \\
Contrast volume $(\mathrm{mL})$ & $64.7 \pm 18.6$ \\
Bleeding $(\mathrm{mL})$ & $410 \pm 218.1$ \\
No. stent grafts & \\
2 & 16 \\
3 & 1 \\
Technical success & 17 \\
No. distal run-offs & \\
2 & 14 \\
1 & 3 \\
BTK intervention & 13 \\
Iliac stenting & 5 \\
\hline
\end{tabular}

BTK, below the knee. 
patient had symptom recurrence because of thrombosis of the Viabahn stent graft, and open thrombectomy was performed on this patient. The ABI increased from $0.51 \pm 0.10$ preoperatively to $1.00 \pm 0.34$ at 12 months $(\mathrm{P}=0.001)$, and $1.00 \pm 0.50$ at 24 months $(\mathrm{P}=0.003)$ postoperatively. The primary (Figure 3A) and secondary patency rates (Figure 3B) at 12 months were both $88.2 \%$, and at 24 months they were $69.2 \%$ and $84.6 \%$, respectively.

\section{Discussion}

Surgical bypass is an effective treatment for severe atherosclerotic lower extremity arterial occlusive disease. This treatment, however, is not always possible because of an absence of suitable veins, lack of an appropriate distal target, or difficulty in providing vascular control or in clamping of the SFA for revascularization due to severe calcification. Because of recent advancements in technology and the favorable outcomes of EVT, an increasing number of physicians now use an endovascular-first strategy to treat challenging SFA occlusive lesions that were previously deemed unsuitable for EVT. The technical success rate in treating TASC C or D FP arterial occlusive disease is approximately $90-100 \% .^{1-3}$ The most common cause of failure is an inability to re-enter the distal true lumen. Some of these patients can be treated using the SAFARI technique or re-entry devices. SAFARI, however, does not necessarily work in difficult cases, and the re-entry device is expensive and not available in every country. VAB requires only a $3-\mathrm{cm}-$ long skin incision and a small arteriotomy for retrieving both the antegrade and retrograde guidewires. The technical success was $100 \%$ in the present 17 patients. We believe that VAB is an alternative hybrid treatment for PAD when conventional EVT fails, and when patients are not eligible for bypass surgery.

The VIPER trial reported a 73\% 12-month primary patency rate in patients with long FP steno-occlusive lesions treated using Viabahn endoprosthesis with heparin bioactive surfaces; $56 \%$ of the lesions were occlusions, the mean lesion length was $19 \mathrm{~cm}$, and $60 \%$ of the enrolled

\begin{tabular}{|lc|}
\hline Table 3. Clinical Results & $\begin{array}{c}\text { VAB } \\
(\mathbf{n = 1 7 )}\end{array}$ \\
Procedure complication & $1(5.88)$ \\
Death & $2(11.76)$ \\
PAD-related death & $1(5.88)$ \\
Major amputation & $2(11.76)$ \\
Acute MI & $3(17.65)$ \\
Stroke & $0(0)$ \\
New hemodialysis & $1(5.88)$ \\
TLR & $4(23.53)$ \\
Edge stenosis & $3(17.65)$ \\
ABI & \\
12 months & $1.00 \pm 0.34$ \\
24 months & $1.00 \pm 0.50$ \\
1-year primary patency rate & 88.2 \\
1-year secondary patency rate & 88.2 \\
2-year primary patency rate & 69.2 \\
2-year secondary patency rate & 84.6 \\
\hline
\end{tabular}

Data given as mean $\pm S D, n(\%)$ or $\%$. $A B I$, ankle-brachial index; $\mathrm{MI}$, myocardial infarction; PAD, peripheral arterial disease; TLR, target lesion revascularization; VAB, Viabahn-assisted bypass.

limbs had TASC II C or D lesions. ${ }^{6}$ The VIASTAR group also reported that the intention-to-treat primary patency rate at 12 months was $70.9 \%$ in patients with symptomatic FP steno-occlusive disease; the mean lesion length was $19.3 \mathrm{~cm} .{ }^{5}$ In the present VAB group, the mean SFA occlusive length was $19.7 \mathrm{~cm}$, and all the limbs had TASC II C or $\mathrm{D}$ lesions. The 12-month primary patency rate was $88.2 \%$, which is superior to that reported from those studies. We believe that if a few rules - such as correct graft sizing; extending the device to relatively disease-free areas of the vessel; and not performing angioplasty beyond the area of planned stent coverage to avoid edge stenosis ${ }^{\mathbf{7}, 8}$ are strictly followed, VAB is a feasible alternative treat-
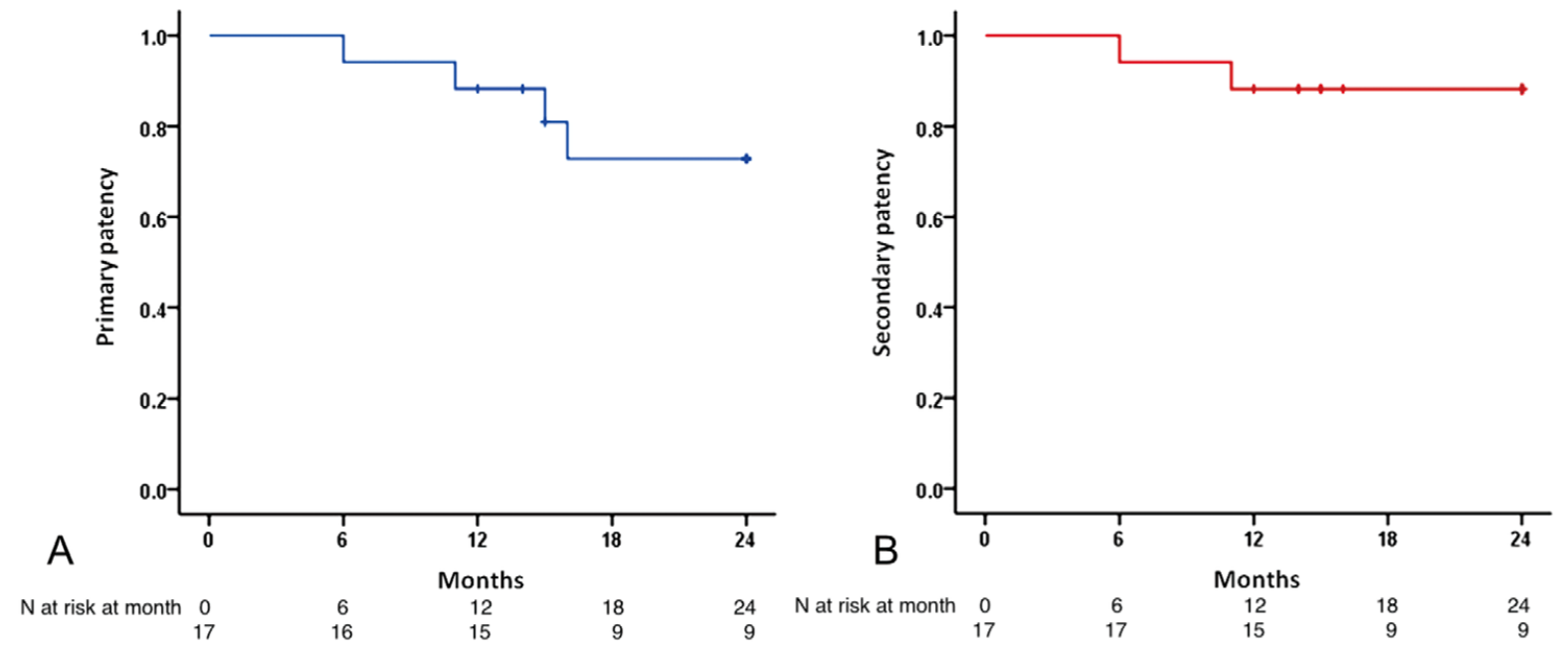

Figure 3. Kaplan-Meier curves of $(\mathbf{A})$ primary patency rate and $(\mathbf{B})$ secondary patency rate during follow-up. 
ment for long SFA steno-occlusive lesions.

The use of GSV bypass remains the gold standard for surgical revascularization of PAD, when available. The alternative conduit is prosthetic polytetrafluoroethylene (PTFE). The primary patency rates at 12 and 24 months for above-the-knee FP bypass using GSV or PTFE graft are around $83 \%, 80 \%$ and $74 \%, 60 \%$ respectively. ${ }^{12}$ The primary patency in the present VAB group was superior to the PTFE bypass graft. Also, VAB requires only a $3-\mathrm{cm}$ incision above the middle SFA, which can be performed under local anesthesia. The avoidance of general anesthesia and multiple incision wounds may facilitate recovery and shorten the length of hospital stay.

Some technical steps should be followed when using the VAB technique. First, the arteriotomy (skin incision) site should be located away from the popliteal artery because it is deeper than the SFA and may require a relatively large incision for enabling exposure. The middle of the SFA is an ideal site for the arteriotomy to retrieve the antegrade and retrograde guidewires because it is relatively superficial and easily accessible. Second, the proximal and distal landing zones of the Viabahn stent graft should be longer than the normally used 1-cm zones because a short segment of the Viabahn is outside the native SFA. When the patient performs daily activities, the movement of the endoprosthesis may become more prominent than usual. Thus, we recommend that the landing zone be at least $3 \mathrm{~cm}$ away from the arteriotomy site in the disease-free SFA to prevent the stent graft from exiting the SFA. Third, while performing pre-dilatation from the antegrade access, finger compression of the arteriotomy site is necessary after deflation of the balloon to prevent bleeding. Most blood loss occurs at this stage. After deployment of the Viabahn stent graft, no further bleeding problems are encountered. Finally, skin incisions near pre-existing ulcers or infection of the medial thigh should be avoided because this increases the risk of wound complication.

\section{Study Limitations}

The limitations of this study were its single-center and retrospective design, in which treatment was allocated according to surgeon preference. We cannot draw conclusions regarding the superiority of VAB compared with other treatments. The small sample size might have introduced bias. Additional studies are warranted to evaluate the long-term outcomes of this novel technique.

\section{Conclusions}

$\mathrm{VAB}$ is a safe alternative procedure that does not require special or expensive devices; it requires only a small skin incision. The short-term and midterm results are acceptable for treating TASC II C or D FP occlusive lesions, but additional studies are warranted to evaluate the long-term outcomes.

\section{Funding}

This research did not receive any specific grant from funding agencies in the public, commercial, or not-for-profit sectors.

\section{Acknowledgment}

We thank the Taiwan Association of Cardiovascular Surgery Research for their assistance.

\section{Disclosures}

The authors declare no conflicts of interest.

\section{References}

1. Saxon RR, Coffman JM, Gooding JM, Ponec DJ. Long-term patency and clinical outcome of the Viabahn stent-graft for femoropopliteal artery obstructions. J Vasc Interv Radiol 2007; 18: $1341-1350$

2. Geraghty PJ, Mewissen MW, Jaff MR, Ansel GM; for the VIBRANT Investigators. Three-year results of the VIBRANT trial of VIABAHN endoprosthesis versus bare nitinol stent implantation for complex superficial femoral artery occlusive disease. J Vasc Surg 2013; 58: 386-395.

3. Kim SJ, Kim W, Kim JB, Hong MJ, Kang WY, Hwang SH, et al. Determinants of procedural success and patency following subintimal angioplasty in patients with TASC C and D femoropopliteal arterial disease. Circ J 2010; 74: 1959-1964.

4. Norgren L, Hiatt WR, Dormandy JA, Nehler MR, Harris KA, Fowkes FGR; on behalf of the TASC II Working Group. Inter-Society consensus for the management of peripheral arterial disease (TASC II). J Vasc Surg 2007; 45(Suppl): S5-S67.

5. Lammer J, Zeller T, Hausegger KA, Schaefer PJ, Gschwendtner M, Mueller-Huelsbeck S, et al. Heparin-bonded covered stents versus bare metal stents for complex femoro-popliteal artery lesions: The randomized VIASTAR trial. J Am Coll Cardiol 2013; 62: $1320-1327$.

6. Saxon RR, Chervu A, Jones PA, Bajwa TK, Gable DR, Soukas PA, et al. Heparin-bonded, expanded polytetrafluoroethylene-lined stent graft in the treatment of femoropopliteal artery disease: 1-year results of the VIPER (Viabahn Endoprosthesis with Heparin Bioactive Surface in the Treatment of Superficial Femoral Artery Obstructive Disease) trial. J Vasc Interv Radiol 2013; 24: $165-173$.

7. Ohki T, Kichikawa K, Yokoi H, Uematsu M, Yamaoka T, Maeda K, et al. Outcomes of the Japanese multicenter Viabahn trial of endovascular stent grafting for superficial femoral artery lesions. J Vasc Surg 2017; 66: 130-142.

8. Kedora J, Hohmann S, Garrett W, Munschaur C, Theune B, Gable D. Randomized comparison of percutaneous Viabahn stent grafts vs. prosthetic femoral-popliteal bypass in the treatment of superficial femoral arterial occlusive disease. J Vasc Surg 2007; 45: 10-16.

9. Aihara H, Soga Y, Mii S, Okazaki J, Yamaoka T, Kamoi D, et al. Comparison of long-term outcome after endovascular therapy versus bypass surgery in claudication patients with Trans-Atlantic Inter-Society Consensus-II C and D femoropopliteal disease. Circ J 2014; 78: $457-464$.

10. Spinosa DJ, Hathun NL, Bissonette EA, Cage D, Leung DA, Angle JF, et al. Subintimal arterial flossing with antegraderetrograde intervention (SAFARI) for subintimal recanalization to treat chronic critical limb ischemia. J Vasc Interv Radiol 2005; 16: $37-44$

11. Lin TC, Chen PL, Chen IM. A novel hybrid Viabahn-assisted bypass technique: Extra-arterial flossing wiring for chronic total occlusion in superficial femoral artery. EJVES Short Rep 2016; 32: $14-17$

12. Shishehbor MH, White CJ, Gray BH, Menard MT, Lookstein $\mathrm{R}$, Rosenfield K, et al. Critical limb ischemia: An expert statement. J Am Coll Cardiol 2016; 68: 2002-2015. 